\title{
Revisão integrativa de estudos no âmbito da psicologia sobre as representações sociais da imagem corporal: o que dizem as produções acadêmicas
}

\author{
Integrative review of studies in the field of psychology on the social representations of body image: \\ what academic productions say \\ Revisión integradora de estudios en el campo de la psicología sobre las representaciones sociales de \\ la imagen corporal: lo que dicen las producciones académicas
}

\author{
Alana Ribeiro Dutra \\ ORCID: https://orcid.org/0000-0002-8002-3864 \\ UNIBRAS - Faculdade de Quatro Marcos, Brasil \\ E-mail: alana_dutra@outlook.com \\ Angélica da Silva Rangel Gonçalves \\ ORCID: https://orcid.org/0000-0002-4697-5845 \\ UNIBRAS - Faculdade de Quatro Marcos, Brasil \\ E-mail: angelicadsrg@gmail.com \\ Jeysson Ricardo Fernandes da Cunha \\ ORCID: https://orcid.org/0000-0002-9688-2313 \\ UNIBRAS - Faculdade de Quatro Marcos, Brasil \\ E-mail: jeyssonrf10@gmail.com
}

\begin{abstract}
Resumo
O presente estudo aborda a importância dos conceitos da representação social que abrange o corpo e a percepção da imagem deste no contexto da contemporaneidade. O objetivo principal é analisar a percepção da imagem corporal e sua influência na saúde biopsicossocial, de acordo com as subjetividades de cada sujeito e as influências midiáticas exercidas por cada sociedade de modo geracional. Este estudo corresponde a um estudo bibliográfico, realizado através das plataformas formal do Catálogo de Teses e Dissertações (CAPES), Google Acadêmico e Biblioteca Digital de Teses e Dissertações (BDTD), utilizando para pesquisa o total de seis artigos, para o resultado e discussão. A partir do pressuposto as representações sociais fazem parte do contexto de vida de todo e qualquer individuo que esteja inserido em determinada sociedade, na qual a relação que a mídia exerce sobre os corpos produz conteúdos subjetivos tem como função balizar o corpo de modo a determinar padrões estéticos em que os sujeitos orientam suas atitudes e comportamentos diante de si e do outro. Os dados revelam que a percepção da imagem corporal está ligada a conteúdos representacionais que fazem parte de uma construção social, no qual o sujeito encontra-se disposto a seguir padrões e regras de acordo com o meio em que está inserido. Tem-se, então, uma tênue distinção entre beleza e saúde que desconsidera os efeitos negativos do corpo que, por sua vez, podem levar ao crescimento de sujeitos com transtornos relacionados a imagem corporal.
\end{abstract}

Palavras-chave: Psicologia; Representação social; Imagem corporal.

\begin{abstract}
The present study addresses the importance of the concepts of social representation that encompasses the body and the perception of the body's image in the context of contemporaneity. The main objective is to analyze the perception of body image and its influence on biopsychosocial health, according to the subjectivities of each subject and the media influences exerted by each society in a generational way. This study corresponds to a bibliographic study, carried out through the formal platforms of the Catalog of Theses and Dissertations (CAPES), Google Scholar and Digital Library of Theses and Dissertations (BDTD), using a total of six articles for research, for the result and discussion . Based on the assumption, social representations are part of the life context of each and every individual who is inserted in a certain society, in which the relationship that the media exercises over bodies produces subjective content has the function of guiding the body in order to determine patterns esthetics in which the subjects guide their attitudes and behaviors towards themselves and the other. The data reveal that the perception of body image is linked to representational content that is part of a social construction, in which the subject is willing to follow standards and rules according to the environment in which he is inserted. There is, then, a fine distinction between beauty and health that disregards the negative effects of the body, which, in turn, can lead to the growth of subjects with disorders related to body image.
\end{abstract}

Keywords: Psychology; Social representation; Body image. 


\begin{abstract}
Resumen
El presente estudio aborda la importancia de los conceptos de representación social que engloba el cuerpo y la percepción de la imagen del cuerpo en el contexto de la contemporaneidad. El objetivo principal es analizar la percepción de la imagen corporal y su influencia en la salud biopsicosocial, según las subjetividades de cada sujeto y las influencias mediáticas que cada sociedad ejerce de manera generacional. Este estudio corresponde a un estudio bibliográfico, realizado a través de las plataformas formales del Catálogo de Tesis y Disertaciones (CAPES), Google Scholar y Biblioteca Digital de Tesis y Disertaciones (BDTD), utilizando un total de seis artículos de investigación, para el resultado y discusión. A partir del supuesto, las representaciones sociales forman parte del contexto de vida de todos y cada uno de los individuos que se insertan en una determinada sociedad, en la que la relación que los medios ejercen sobre los cuerpos produce un contenido subjetivo que tiene la función de guiar el cuerpo para determinar Patrones de estética en los que los sujetos orientan sus actitudes y comportamientos hacia sí mismos y hacia el otro. Los datos revelan que la percepción de la imagen corporal está ligada a un contenido representacional que forma parte de una construcción social, en la que el sujeto está dispuesto a seguir estándares y reglas de acuerdo con el entorno en el que se inserta. Existe, entonces, una fina distinción entre belleza y salud que desconoce los efectos negativos del cuerpo, lo que, a su vez, puede conducir al crecimiento de sujetos con trastornos relacionados con la imagen corporal.
\end{abstract}

Palabras clave: Psicología; Representación social; Imagen corporal.

\title{
1. Introdução
}

$\mathrm{Na}$ atualidade nos deparamos com um conjunto de conhecimentos socialmente elaborados que precisam ser estudados e discutidos, ao qual estão na maioria das vezes ligados aos conceitos sobre o sujeito e as coisas que o cerca, diante disso, é inevitável abordar as representações sociais que está presente na vida dos sujeitos enquanto seres pertencentes de uma sociedade, seja os mesmos se posicionando de modo individual ou de maneira coletiva, podendo ser um meio em que há a descoberta da percepção de si ou do outro, o que permite a descoberta da subjetividade dos envolvidos num grupo social (Almeida, Santos \& Araujo, 2014).

Desta forma, este estudo intenta responder ao seguinte problema de pesquisa: quais são as representações sociais do corpo presentes na sociedade e como estas podem influenciar de maneira gradativa os sujeitos na contemporaneidade?

Neste sentido, o corpo e a imagem construída através dele é construído e significado socialmente e está relacionado com os comportamentos dos sujeitos que buscam mostrar suas qualidades em meio aos efeitos com base no que é socialmente aceitável e, assim procuram reproduzir ou se espelhar em outros grupos. A partir disso acabam por exagerar no modo de ver o seu próprio corpo como algo objetificado e, assim, implicando essas concepções em atos que podem gerar graves problemas psicológicos, atos estes que competem a psicologia estudar e compreender, pois integra um conjunto de fenômenos mediante as relações humanas, conduta que a psicologia social está inserida, que atribui encaixar um significado diferente que são observáveis, promovendo uma mudança constante e transformação enquanto ao sujeito que se diferencia em suas concepções.

Sendo assim, esse estudo aborda, a partir de uma revisão integrativa, as representações sociais do corpo e as consequências da mídia na objetificação e na saúde biopsicossocial. Compreende-se, assim, o corpo como um fenômeno histórico e social, cujas representações compartilhadas refletem a cultura e modo que determinados grupos percebem o que é aceitável ou não dentro dos padrões estabelecidos na relação Eu-Outro.

Desta forma, a origem da teoria das representações sociais é uma tendência da psicologia social que remonta as formulações feitas por Durkheim diante o conceito de Representações Coletivas. Partindo dessa concepção Moscovici (1978) introduz uma nova modalidade de conhecimento social baseada no senso comum denominada de representações sociais. Logo, as representações sociais podem ser compreendidas enquanto uma maneira específica de compreender e comunicar o que já sabemos (Moscovici, 2007) de maneira que faça sentido a um determinando grupo ou conjunto social. Desta forma,

"Elas sempre possuem duas faces, que são interdependentes, como duas faces de uma folha de papel: a face icônica e a face simbólica. Nós sabemos que: representação = imagem/significação; em outras palavras, a representação iguala toda imagem a uma ideia e toda ideia a uma imagem" (Moscovici, 2001, p. 47). 
Neste pressuposto se desenvolve a teoria das representações sociais por Moscovici (1961/1976), sendo este relatado em La Psicanalyse: Son image et son public. Esta teoria embasa-se no fundamento de um estudo científico-social da comunicação possuindo uma interrelação direta com as representações sociais e o senso comum desde sujeitos a grupos (Almeida, Santos \& Trindade, 2014).

No que se refere a definição da representação social há uma variedade de definições na qual autores demonstram a amplitude da teoria (Boas, 2004), as quais estão evidenciadas a seguir: “[...] é uma forma de conhecimento, socialmente elaborada e partilhada, com um objetivo prático, e que contribui para a construção de uma realidade comum a um conjunto social" (Jodelet, 2001, p.22).

Já para Spink (1993, p. 300) a representação social:

"São, consequentemente, formas de conhecimento que se manifestam como elementos cognitivos - imagens, conceitos, categorias, teorias - , mas que não se reduzem jamais aos componentes cognitivos. Sendo socialmente elaboradas e compartilhadas, contribuem para a construção de uma realidade comum, que possibilita a comunicação. Deste modo, as representações são, essencialmente, fenômenos sociais que, mesmo acessados a partir do seu conteúdo cognitivo, têm de ser entendidos a partir do seu contexto de produção. Ou seja, a partir das funções simbólicas e ideológicas a que servem e das formas de comunicação onde circulam”.

De acordo com Abric (1998 apud Camargo, 2011) as representações tem um papel essencial nas relações de caráter tanto social quanto prático e apontam quatro funções fundamentais: a) Função de saber, que permite adquirir conhecimentos de modo coerente diante dos valores por parte dos sujeitos; b) Função identitária, que representa aspectos do grupo onde está inserido; c) Função de orientação, é utilizado como modelo de direção na ação; d) Função justificadora, na qual o sujeito precisa justificar suas decisões e comportamentos.

No que se refere a formação das representações sociais há dois processos de pensamento pertinentes que se interligam na geração das representações sociais, sendo estes objetivação e ancoragem. Estes dois processos cognitivos são baseados pela memória e em conclusões passadas na qual possibilita tornar comum algo que seja estranho e inominável por um grupo. Assim, a ancoragem é um processo cujo algo ameaçador e desconhecido ao grupo se torna classificável e nomeável. A objetivação, por sua vez, produz a cristalização de um conceito/ideia, isto é, tornando o abstrato em algo concreto (Moscovici, 2007).

Vale ressaltar que de acordo com Moscovici (1978, p. 41), "as relações sociais que estabelecemos no cotidiano são frutos de representações que são facilmente apreendidas". Ainda segundo o autor, a representação social possui uma dupla dimensão, Sujeito e Sociedade, e situa-se no limiar de uma série de conceitos sociológicos e psicológicos.

Deste modo as representações sociais se tratam de fenômenos observáveis, pois são sempre de algo ou de alguma coisa, ou seja, pessoas e/ou objetos, inclusive no que se refere a trabalho científico. Nos dias atuais estes fenômenos vêm tomando pauta nas áreas das ciências humanas, que é o caso da Psicologia, pois esta forma de observá-lo acontece de modo completamente natural, e com isso acabam por se proliferar nas redes, sejam elas no discurso, mensagem e nos veículos midiáticos (Jodelet, 2001).

Pensando o corpo enquanto objeto social, tem-se que a conceituação do corpo tomado como componente constituído e constituinte de representações sociais, envolve diversas características que vão desde a aparência física, estado biológico, construção social, natural e cultural, a partir de uma perspectiva que faz parte da história das civilizações (Rosário, 2006).

"As concepções e as representações do corpo, bem como a beleza, sofreram transformações ao longo da história em cada sociedade, associadas as mudanças socioeconômicas e culturais. Assim, conhecer como determinado grupo pensa e concebe o corpo pode contribuir para compreender a hegemonia de uma estética corporal, como também esclarece a amplitude dos significados relativos ao próprio corpo" (Braga, Molina \& Figueiredo, 2008, p. 88). 
Deste modo, a história do corpo humano se remete substancialmente a história das civilizações, no qual se designa as regras, costumes e valores do sujeito em relação ao ambiente que o cerca. Assim o corpo passa por evoluções ao longo de eras, isto acontece de acordo com a sociedade ao qual está inserido (Barbosa, Mattos \& Costa, 2011).

$\mathrm{Na}$ Grécia antiga, a imagem do corpo era vista de maneira com que corpo e espírito estivessem em harmonia, sendo o corpo submetido a exercícios físicos e a mente por meditação. O corpo nu, era visto com apreço e sinal de saúde e beleza, de modo que a moral entre corpo e sexo não possuía autoritarismo e rigidez, porém estes estavam estritos apenas a "cidadãos". Já no Cristianismo, o corpo passa a ser "proibido", uma fonte de pecado, contudo por outro lado também é glorificado diante o corpo sofredor de Cristo. Em relação a idade média, o corpo volta a ser instrumento da representação social, mesmo que regido ainda pelo cristianismo sob a concepção de corpo e alma. A partir da Era Moderna com o renascimento, prevalece sobre o corpo a cientificidade, as atividades físicas agora passam por um sistema de regras que visam a saúde corporal, no entanto com o capitalismo o corpo passou-se a ser visto como uma máquina sob a produção industrial - apontam Barbosa, Matos \& Costa (2011).

Nos dias atuais, pode-se dizer que o corpo é tido como um objeto, a indústria da beleza se mostra cada vez maior, e os sujeitos cada vez mais interessados em seus produtos, investindo em seus corpos para fins de prazer, sensualismo e estimulação social (Barbosa, Matos \& Costa, 2011).

Deste modo, Tavares (2003) diz que a compreensão do conceito de Imagem Corporal está vinculada ao significado dos termos imagens e corpo e que sua definição não é simplesmente uma questão de linguagem, tem uma dimensão muito maior, se pensarmos na subjetividade de cada indivíduo.

As pessoas aprendem a avaliar seus corpos através da interação com o ambiente, assim sua autoimagem é desenvolvida e reavaliada continuamente durante a vida inteira (Becker, 1999), mas as necessidades do contexto social acabam se sobrepondo as de caráter individual. Somos pressionados em numerosas circunstâncias a concretizar, em nosso corpo, o corpo ideal da nossa cultura. De acordo com Vargas (2014):

\footnotetext{
"Atualmente, muitos setores da sociedade, principalmente a mídia, deixam explícitas discussões acerca do corpo e padrões estéticos que valorizam o corpo perfeito, com referência em um único padrão. [...] O sentimento da busca pela modificação do corpo vem perseguindo os indivíduos, levando-os a utilizar medidas que podem ser prejudiciais à saúde, tais como o uso de anorexígenos e anabolizantes, tratamentos estéticos variados e práticas como a indução de vômitos, jejuns prolongados, dietas de restrição, a fim de se obter resultado "milagroso" em curto espaço de tempo" (Vargas 2014, p.74).
}

Com a adoção destes métodos prejudiciais à saúde mencionados, o indivíduo tende a desencadear alguns transtornos patológicos durante o percurso de sua vida, ao qual alguns destes são classificados no DSM-5 (2014) Anorexia Nervosa, sendo uma restrição alimentar evitando alimentos em uma tentativa de diminuição do peso corporal, Bulimia Nervosa, no qual o indivíduo evite alimentos, porém sofre de uma compulsão que ao ingerir uma grande quantidade de alimentos de uma só vez, se sente culpado e induz ao vômito, Transtorno Dismórfico Corporal, ocorre uma distorção da própria imagem devido uma grande preocupação com a mesma.

Nesse sentido, a busca de um corpo ideal no ápice de exaltação acabam por fortalecer as representações sociais do corpo, pois ela acontece diante as trocas coletivas e individuais de experiências através da vivencia com o outro, por meio da cultura e dos próprios conceitos, estas efetuadas mediante o corpo que acarreta em decorrência dos conhecimentos socialmente elaborados pela sociedade (Jodelet, 2001). Portanto o corpo pode ser compreendido como fluxo de representações sociais que o indivíduo auto institui (Novikoff, 2014). 


\section{Metodologia}

A metodologia adotada caracteriza este estudo como qualitativo que, de acordo com Denzin e Lincoln (2006), envolve uma abordagem interpretativa do mundo, o que significa que seus pesquisadores estudam as coisas em seus cenários naturais, tentando entender os fenômenos em termos dos significados que as pessoas a eles conferem; faz similaridade ao problema de pesquisa envolvendo o fenômeno existente na percepção da imagem corporal de modo histórico, simbólico e social que é apresentado entre o sujeito e o mundo ao qual está introduzido, mediante um estudo bibliográfico focado na investigação do objeto "corpo" no contexto das representações sociais em processos de avaliação da produção científica apresentada em forma de artigos.

Assim, optou-se pela revisão integrativa, que tem como característica proporcionar através de um método de pesquisa um levantamento de dados sobre estudos bibliográficos, que para Botelho et, al. (2011), este é um método mais amplo que permite para os autores compreender os fenômenos a partir da revisão dos conhecimentos de trabalhos e pesquisas científicas já elaborados, podendo assim possibilitar novos estudos que são pautados em etapas a serem seguidas que vão de acordo com o resultado de cada autor.

Na organização deste estudo, entre as várias técnicas de investigação dos conteúdos das representações sociais, optouse pela análise de conteúdo temática. Neste sentido, a análise de conteúdo tem uma dimensão descritiva que visa dar conta do que nos foi narrado e uma dimensão interpretativa que decorre das interrogações do analista em face de um objeto de estudo, com recurso a um sistema de conceitos teórico-analíticos cuja articulação permite formular inferências (Guerra, 2006).

A seleção dos artigos para a produção se deu a partir das plataformas de dados mediante fontes de pesquisa sobre bancos de teses e dissertações da CAPES ${ }^{1}$, Google Acadêmico² e Biblioteca Digital de Teses e Dissertações - BDTD ${ }^{3}$, disponíveis na rede de internet sob o expositor: Representação social do corpo. Deste modo utilizando a revisão integrativa como base de estudo, o intervalo de tempo da pesquisa percorre aproximadamente 4 meses agosto/dezembro, a partir disso foi possível realizar o levantamento dos dados e resultados encontrados com a pesquisa diante os estudos científicos que diretamente são ligados ao contexto da psicologia. A escolha desta modalidade de pesquisa ocorre apoiado na necessidade de entender e interpretar os estudos bibliográficos em concordância ao tema desejado.

\section{Resultados e Discussão}

Com base em uma pesquisa bibliográfica nos bancos de teses e dissertações do CAPES e BDTD, foi possível um levantamento de dados de pesquisa a requisito de mestrado e doutorado que possibilitam discussões e análises diante deste assunto. Os dados resultaram em uma sondagem a partir do descritor Representação Social do Corpo que possibilitou os seguintes fins numéricos: Banco Capes- 1.980, no Google Acadêmico o resultado geral da pesquisa formou-se aproximadamente em 472.000 resultados, em relação ao banco de teses e dissertações BDTD- 579.

Neste caso o descritor passa por alteração em uma tentativa de redução do número expressivo de dados encontrados, devido preocupação em abordar de modo mais específico a área da Psicologia, pois é o campo que representa todo o contexto ao qual este estudo está sendo construído. Em geral, o corpo possui influência ao convívio de outros sujeitos, podendo ser compreendido a partir de funções sociais, que se mostram videntes e presentes nas relações com o meio social.

Este estudo priorizou-se em sua seleção, análise e discussões de artigos que apoiam o escopo da representação social, ancorados com o objeto corpo, de acordo com a Tabela 1.

\footnotetext{
${ }^{1}$ CAPES Periódico > https://www-periodicos-capes-gov-br.ezl.periodicos.capes.gov.br/index.php?

${ }^{2}$ Google Acadêmico > https://scholar.google.com.br/?hl=pt

${ }^{3}$ Biblioteca Digital de Teses e Dissertações - BDTD > https://bdtd.ibict.br/vufind/
} 
Tabela 1. Estudos selecionados.

\begin{tabular}{|c|c|c|c|c|c|}
\hline \multicolumn{6}{|c|}{$\begin{array}{c}\text { BANCO DE TESES E DISSERTAÇÕES: CAPES, Google Acadêmico e Biblioteca Digital de Teses e } \\
\text { Dissertações (BDTD) } \\
\text { Descritor: Representação Social do Corpo }\end{array}$} \\
\hline Ano & Plataforma & $\begin{array}{c}\text { Modalidad } \\
\text { e }\end{array}$ & Autor & Título & Universidade \\
\hline 2014 & CAPES & Artigo & $\begin{array}{c}\text { Justo; Camargo } \\
\text { \& Alves }\end{array}$ & $\begin{array}{l}\text { Os efeitos de contexto } \\
\text { nas representações } \\
\text { sociais sobre o corpo }\end{array}$ & $\begin{array}{c}\text { Universidade Federal } \\
\text { de Santa Catarina }\end{array}$ \\
\hline 2011 & CAPES & Artigo & $\begin{array}{c}\text { Camargo; Justo } \\
\text { \& Alves }\end{array}$ & $\begin{array}{l}\text { As funções sociais e as } \\
\text { representações sociais } \\
\text { em relação ao corpo: } \\
\text { uma comparação } \\
\text { geracional }\end{array}$ & $\begin{array}{l}\text { LACCOS- } \\
\text { Universidade Federal } \\
\text { de Santa Catarina }\end{array}$ \\
\hline 2009 & $\begin{array}{c}\text { Google } \\
\text { Acadêmico }\end{array}$ & Artigo & $\begin{array}{l}\text { Secchi; } \\
\text { Camargo \& } \\
\text { Bertoldo }\end{array}$ & $\begin{array}{l}\text { Percepção da imagem } \\
\text { corporal e } \\
\text { representações sociais } \\
\text { do corpo }\end{array}$ & $\begin{array}{c}\text { Universidade do } \\
\text { Planalto Catarinense }\end{array}$ \\
\hline 2008 & $\begin{array}{c}\text { Google } \\
\text { Acadêmico }\end{array}$ & Artigo & $\begin{array}{l}\text { Goetz; } \\
\text { Camargo; } \\
\text { Bertoldo \& } \\
\text { Justo }\end{array}$ & $\begin{array}{c}\text { Representação Social } \\
\text { do corpo na mídia } \\
\text { impressa }\end{array}$ & $\begin{array}{c}\text { Universidade Federal } \\
\text { de santa Catarina }\end{array}$ \\
\hline 2009 & BDTD & Tese & Furtado & $\begin{array}{l}\text { Representação social do } \\
\text { corpo, mídia e atitudes }\end{array}$ & $\begin{array}{c}\text { Universidade Federal } \\
\text { de Santa Catarina }\end{array}$ \\
\hline 2011 & BDTD & Dissertação & Justo & $\begin{array}{c}\text { Representações sociais } \\
\text { sobre o corpo e } \\
\text { implicações do } \\
\text { contexto de inserção } \\
\text { deste objeto }\end{array}$ & $\begin{array}{c}\text { Universidade Federal } \\
\text { de Santa Catarina }\end{array}$ \\
\hline
\end{tabular}

Fonte: Levantamento da pesquisa.

Conforme o exposto da Tabela 1, a temática gira em torno do âmbito das representações sociais o que possibilita uma vasta gama de discussões no cenário entre sociedade e sujeito.

O estudo de Justo, Camargo e Alves (2014) busca compreender a relação existente entre representação social e corpo, objetivando o papel existente entre ambos, que se trata de um quase experimental, no qual participaram da pesquisa 79 participantes distribuídos de modo uniforme entre homens e mulheres, jovens e adultos, sendo integrantes da Universidade Federal de Santa Catarina - UFSC, envolvendo como métodos um roteiro de grupo-focal e questionário. Verificou-se que a saúde é a base estruturante da representação social RS, assim é difícil pensar em corpo, sem pensar na saúde do corpo. Os resultados apresentaram-se em dois contextos, um de saúde e o outro de beleza diante o corpo, assim a maioria dos participantes estão saudáveis em relação ao peso e satisfeitos com a imagem corporal, porém não houve uma diferença relevante entre os sexos, mais sim entre os grupos geracionais.

No que diz respeito a pesquisa de Camargo, Justo e Alves (2011), partem do princípio de representação individual e social, ao qual possui em sua temática uma comparação gestacional, sendo estudado estas em dois grupos em um total de 79 participantes de maneira equivalente entre homens, mulheres jovens e adultos da Universidade Federal de Santa Catarina UFSC, se dedicando a interpelação entre representação social, funções sociais e o corpo. Neste se destaca que as diferenças e mudanças geracionais são mais relevantes do que a existentes entre os sexos diante a conclusão de que o corpo humano é um organismo natural. Os resultados apontam que tanto a conexão social quanto individual e a aparência do corpo fazem parte da personalidade do indivíduo, e que estes demonstram não considerar a opinião dos outros.

Secchi, Camargo e Bertoldo (2009) traz em sua delimitação a percepção da imagem corporal vinculada a teoria das representações sociais objetivando apurar a relação de ambas entre o público de diferentes cursos de universidades de Santa 
Catarina, 278 participantes do sexo feminino dos cursos de Psicologia, Moda e Educação Física. As representações sociais partem do contexto de aparência do corpo em suas relações pessoais, a relevância entre beleza e saúde ligados ao corpo padronizado e perfeito e a associação dos conceitos anteriores com a capacidade profissional e pessoal que podem levar ao crescimento de indivíduos com transtornos relacionados a imagem corporal. Sendo assim, encontra-se uma contradição das representações sociais com a subjetividade, já que as participantes se mostram insatisfeitas em sua percepção corporal e favoráveis a procedimentos estéticos.

Em relação ao estudo de Goetz et. al. (2008) possui como finalidade pesquisar o campo das representações sociais do corpo ligada a mídia, que se refere a uma pesquisa documental em revistas de beleza e saúde, na qual os dados foram computados através do programa ALCESTE. As representações sociais do corpo são vistas de dois modos, um de carácter físico de acordo com a saúde e estética corporal, e o outro de modo subjetivo do corpo representando uma harmonia física e psicológica.

Furtado (2009) tem como escopo definir as representações sociais de acadêmicos universitários de acordo com a saúde e estética corporal em meio a atitude destes frente a mídia, que se fundamenta na TRS, o qual utiliza-se de dois métodos de pesquisa, um de natureza documental e outra empírica. Assim há uma preocupação com a imagem corporal por parte dos estudantes, o que traz uma atitude negativa, sendo que isto pode se associar a fenômenos históricos sociais e culturais, que visam o sinônimo de magreza como belo, e não privilegiando o conceito de si, de subjetividade e bem-estar.

Para finalizar temos as concepções de Justo (2011) faz uma inter-relação entre representação social do corpo e objeto, tanto que seu objetivo se caracteriza na compreensão do papel em contexto deste objeto diante o corpo sob vastos grupos de dimensões geracionais. Refere-se a uma pesquisa quase experimental, participaram 79 indivíduos entre homens e mulheres, jovens e adultos de uma comunidade universitária, divididos em 16 grupos. Deste modo, o corpo faz parte do contexto ao qual está inserido, possuindo um significado de saúde, sendo também um objeto social que faz parte das vivências desses indivíduos, podendo ser importante possuir uma perspectiva melhor na promoção de qualidade de vida, saúde e bem estar social.

É possível perceber que a continuação entre esses artigos e dos autores que tentaram de alguma forma apresentar a representação social do corpo, com um estudo amplo e que pudesse transmitir vários conhecimentos diante a RS, onde existe o Laboratório LACCOS (Laboratório de Psicologia Social da Comunicação e Cognição) coordenado por Brígido, que envolve as áreas de conhecimento da Psicologia Social e das Representações Sociais, visando assim uma rede de estudo que busca compartilhar todas pesquisas cientificas e ensinos para gerar fontes seguras de conhecimentos.

Nota-se a partir dos artigos encontrados a relevância de uma construção social do corpo, que causa impactos para a vida dos sujeitos, falando de modo geral toda essa discussão gira em torno de como o ser humano como um ser histórico e social buscando sempre estar inserido em um contexto ao qual existem um padrão a ser seguido ou até mesmo compartilhado, nesse meio o corpo é o objeto que está em constante transformação aos olhos da sociedade. Há diversos significados sobre as representações que podem determinar os indivíduos e os grupos em suas escolhas de como se manifestar diante os padrões que a sociedade lhes impõe, o que pode acontecer por tomarem esse padrão como modelo (Jodelet, 2001).

Os autores aqui apresentados constroem uma relação de imagem corporal está ligada à existência de representações sociais ancoradas na cultura e na história com influência no modo de manifestar comportamentos com o viés de busca pela perfeição. Neste prisma, a saúde dos sujeitos e grupos ficam marginalizadas e, com isso, pode acarretar o desenvolvimento de transtornos relacionados a imagem corpo. Nessa perspectiva de estudo o corpo em sua dimensão histórica, é objetivado de acordo com à época, e assim percebe-se que a mídia contribui para a geração de conteúdos representacionais cujo papel central é o de criar uma ideia que seja comum a sujeitos e grupos: o belo = magro; o feio = obeso. Essa é a "atmosfera" que, como anuncia Moscovici (2007), as representações devem ser vistas. 


\section{Considerações Finais}

A partir do desenvolvimento deste estudo foi possível perceber que a imagem corporal, enquanto objeto de estudo, é um campo promissor de estudos. Esta constatação revela a importância deste fenômeno na sociedade, sobretudo, considerando a mídia como fonte de influência social e geradora de representações sociais. Assim, tornou-se possível responder o problema de pesquisa que nos propomos a pensar: quais são as representações sociais do corpo presentes na sociedade e como estas podem influenciar de maneira gradativa os sujeitos na contemporaneidade?

Desta forma, é possível perceber que o corpo é um fenômeno histórico e social, devido os padrões diante a imagem corporal passar por alterações no decorrer de décadas, alterações estas que estão intimamente ligadas ao modo de como os indivíduos de cada sociedade se comportam. Os veículos midiáticos acabam por estimular a distorção da imagem corporal alterando a percepção do corpo, fazendo com que se busque uma imagem perfeita e não real como se existisse um padrão a ser seguido, a busca por este "corpo perfeito" pode acarretar transtornos psicológicos para alcançar a perfeição e o resultado desejável.

Deste modo, o corpo pode ser influenciado devido aos sujeitos buscar padrões a serem seguidos, normalmente acontece pelo desejo de ter o corpo perfeito, neste sentido se espelham em sujeitos que possuem uma alta teoria de fama e beleza, porém esquecem que cada um possui sua subjetividade, ou seja, é um ser único com características e qualidades próprias, assim de qualquer forma são consequenciais querer ser ou submeter seguir um modelo especifico, somente por prazer e fortalecer o próprio ego.

O objetivo central deste estudo foi o interesse em analisar a percepção da imagem corporal e sua influência na saúde biopsicossocial, que desenvolve diante as relações com meio social e sujeitos que influenciam e são influenciados. Assim, a relação que a mídia exerce sobre os corpos produz conteúdos subjetivos cuja função é balizar o corpo de modo a determinar padrões estéticos em que os sujeitos orientam suas atitudes e comportamentos diante de si e do outro.

Nessa perspectiva alcançou-se descobertas de conhecimentos científicos que são valiosos meios de entender como se conduz uma pesquisa, coleta de dados, avaliações dos resultados, mas que também contribui enquanto estudo sobre esse determinado assunto em que envolve as representações sociais e a imagem corporal, onde geralmente podem atingir vários públicos e assim possibilitando a novos estudos na área. Revela-se, assim, a necessidade de estudos que aprofundem a perspectiva da influência na mídia na elaboração de representações sociais e, consequentemente, os impactos disso no cotidiano em relação à saúde mental dos sujeitos. Assim, recomenda-se à pesquisadores que efetuem pesquisas neste sentido em outras partes do Brasil e também com o público infantil.

Por fim, a percepção da imagem corporal está ligada a conteúdos representacionais que fazem parte de uma construção social, no qual o sujeito encontra-se disposto a seguir padrões e regras de acordo com o meio em que está inserido. Tem-se, então, uma tênue distinção entre beleza e saúde que desconsidera os efeitos negativos do corpo que, por sua vez, podem levar ao crescimento de sujeitos com transtornos relacionados a imagem corporal.

\section{Referências}

Almeida, A. M. de O., Santos, M. de F. S., \& Trindade, Z. A. (2014), Teoria das representações sociais 50 anos. (2a ed.), eBook/Pdf.

American Psychiatric Association. (2014). Manual diagnóstico e estatístico de transtornos mentais: Tradução, Maria, I. N. et al, (5a ed.) Artmed.

Barbosa, M. R., Matos, P. M., \& Costa, M. E. (2011). Um olhar sobre o corpo: o corpo ontem e hoje. Psicologia \& Sociedade. 23(1), 2434 https://www.scielo.br/scielo.php?script=sci_arttext\&pid=S0102-71822011000100004

Becker, B. J. (1999). Manual de psicologia aplicada ao exercício e Esporte. Editora Nova Prova.

Boas, L. P. S. V. (2004). Teoria das representações sociais e o conceito de emoção: diálogos possíveis entre Serge Moscovici e Humberto Maturana. Psicologia da Educação Versão Impressa ISSE 1414-6965. http://pepsic.bvsalud.org/scielo.php?script=sci_arttext\&pid=S1414-69752004000200008 
Botelho, L. L. R, Cunha, C. C. A, Macedo, M. (2011) O método da revisão integrativa nos estudos organizacionais. Gestão e Sociedade, 5 (11), $121-136$. https://www.gestaoesociedade.org/gestaoesociedade/article/view/1220

Braga, P. D., Molina, M. C. B. \& Figueiredo, T. A. M. (2008). Representações do corpo: com a palavra um grupo de adolescentes de classes populares. Programa de Pós-Graduação em Atenção à Saúde Coletiva, Centro de Ciência da Saúde, Universidade Federal do Espírito Santo. https://www.scielo.br/scielo.php?pid=S1413-81232010000100014\&script=sci_abstract\&tlng=pt

Camargo, B. V. et al. (2001). Representações sociais do corpo: estética e saúde. (2001). Temas em Psicologia. Vol. 19 n.1 Junho, LACCOS UFSC - Universidade Federal de Santa Catarina, Ribeirão Preto. Apud Abric, J. C. (1998). Abordagem estrutural das representações sociais. Goiânia.

Camargo, B. V, Justo, A. M, Alves, C. D. B. (2011). As funções sociais e as representações sociais em relação ao corpo: uma comparação geracional. Temas em Psicologia. Vol.19 no.1Junho Ribeirão Preto. http://pepsic.bvsalud.org/scielo.php?script=sci_arttext\&pid=S1413-389X2011000100022

Denzin, N. K, Lincoln, Y. S. (2006). Introdução: a disciplina e a prática da pesquisa qualitativa. In: Denzin, N. K. e Lincoln, Y. S. (Orgs.). O planejamento da pesquisa qualitativa: teorias e abordagens. (2a ed.), Artmed.

Furtado, E. R. G. (2009). Representações sociais do corpo, mídia impressa e atitudes. Tese (doutorado) - LACCOS UFSC - Universidade Federal d Santa Catarina, Centro de Filosofia e Ciências Humanas, Programa de Pós-Graduação em Psicologia, Florianópolis.

Goetz, E. R., Camargo, B. V, Bertoldo, R. B, Justo, A M. (2008). Representação social do corpo na mídia impressa. Psicologia \& Sociedade, 20 (2): 226-236, https://www.scielo.br/scielo.php?script=sci_arttext\&pid=S0102-71822008000200010

Guerra, I. C. (2006). Pesquisa qualitativa e análise de conteúdo: sentidos e formas de uso. Principia.

Jodelet, D. (2001) As Representações sociais. Traduzido por Ulup, Lilian. UERJ.

Justo, A. M. (2009). Representações sociais sobre o corpo e implicações do contexto de inserção desse objeto. Dissertação (mestrado) Florianópolis - SC. Universidade Federal de Santa Catarina. https://repositorio.ufsc.br/xmlui/handle/123456789/94981

Justo. A. M, Camargo, B. V, Alves, C. D. B. (2014). Os efeitos de Contexto nas representações sociais sobre o corpo. Psicologia: teoria e pesquisa jul-7sete, vol.30 n.3, ppp.287-297 Universidade Federal de Santa Catarina. https://www.scielo.br/pdf/ptp/v30n3/06.pdf

Moscovici, S. (2007). Representações sociais: investigações em psicologia social: editado em inglês por Gerard Duveen: traduzido do inglês por Pedrinho A. Guareschi. (5a ed.), Vozes.

Moscovici, S. (2001). Das representações coletivas às representações sociais: Elementos para uma história. In D. Jodelet Org. As representações sociais. p.47.

Moscovici, S.(1978). A Representação social da Psicanálise. Tradução Cabral. Rio de Janeiro Zahar, p.41.

Moscovici, S. (2001). As representações sociais um domínio de expansão. In: Jodelet, Denise. (Org)Teoria das Representações Sociais. Tradução Lilian Ulup. EdURJ

Novikoff, C. (2014). Valores, enfrentamento, representações sociais: experiências do ensino superior na área da saúde. Salvador.

Rosário, N. M. Mundo contemporâneo: Corpo em meta-morphose. http://www.comunica.unisinos.br/semiotica/nisia_semiotica/conteudos/corpo.htm

SecchI, K, Camargo, B. V, Bertoldo, R. B. (2009). Percepção da imagem corporal e representações sociais do corpo. Pesq. 25(2). https://www.scielo.br/scielo.php?script=sci_arttext\&pid=S0102-37722009000200011

Spink, M. J. P. (1993). O conceito de representação social na abordagem psicossocial. Cad. Saúde Pública. 9(3). https://www.scielo.br/scielo.php?script=sci_arttext\&pid=S0102-311X1993000300017

Tavares, M. C. (2003) Imagem Corporal: conceito e desenvolvimento. http://www.braspen.com.br/home/wp-content/uploads/2016/12/12-A-influencia-damidia-na-construcao-da-imagem.pdf

Vargas, E. G. A. (2014). A influência da mídia na construção da imagem corporal. Minas Gerais. 